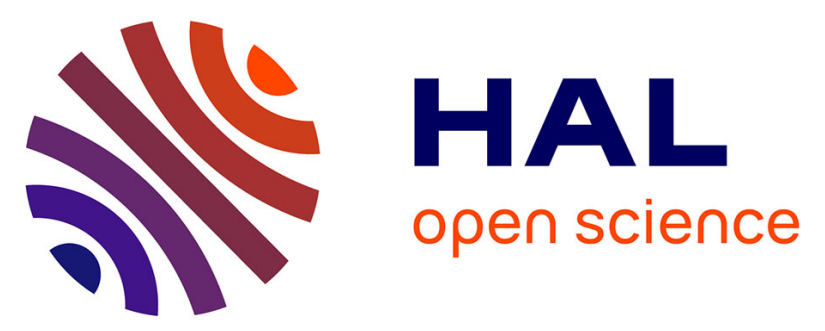

\title{
Interpretation of Pioneer 10 heliospheric Ly? glow data obtained beyond 30 AU
}

\author{
D. L. Judge, V. V. Izmodenov, M. Gruntman, P. Gangopadhyay
}

\section{To cite this version:}

D. L. Judge, V. V. Izmodenov, M. Gruntman, P. Gangopadhyay. Interpretation of Pioneer 10 heliospheric Ly? glow data obtained beyond 30 AU. Astrophysics and Space Sciences Transactions, 2005, 1 (1), pp.29-34. hal-00297472

\section{HAL Id: hal-00297472 \\ https://hal.science/hal-00297472}

Submitted on 9 Dec 2005

HAL is a multi-disciplinary open access archive for the deposit and dissemination of scientific research documents, whether they are published or not. The documents may come from teaching and research institutions in France or abroad, or from public or private research centers.
L'archive ouverte pluridisciplinaire HAL, est destinée au dépôt et à la diffusion de documents scientifiques de niveau recherche, publiés ou non, émanant des établissements d'enseignement et de recherche français ou étrangers, des laboratoires publics ou privés. 


\title{
Interpretation of Pioneer 10 heliospheric Ly $\alpha$ glow data obtained beyond $30 \mathrm{AU}$
}

\author{
D. L. Judge ${ }^{1}$, V. V. Izmodenov ${ }^{2,3,4}$, M. Gruntman ${ }^{1}$, and P. Gangopadhyay ${ }^{1}$ \\ ${ }^{1}$ University of Southern California, Los Angeles, CA 90089, USA \\ ${ }^{2}$ Moscow State University, Department of Aeromechanics and Gas Dynamics, School of Mechanics and Mathematics, \\ Moscow, 119899, Russia \\ ${ }^{3}$ Space Research Institute (IKI), Russian Academy of Sciences \\ ${ }^{4}$ Institute for Problems in Mechanics, Russian Academy of Sciences
}

Received: 2 August 2005 - Revised: 19 October 2005 - Accepted: 24 October 2005 - Published: 9 December 2005

\begin{abstract}
The Pioneer 10 (P10) Ly $\alpha$ dataset is the only dataset that is available for the study of the very local interstellar medium (VLISM) in the downstream direction relative to the incoming interstellar neutral hydrogen flow, at very large distances from the sun. Selected P10 data obtained in 1984 and 1986 at distances between 31.81 to 32.25 and 37.29 to $37.74 \mathrm{AU}$ have been used to estimate the local interstellar neutral hydrogen and proton densities. State of the art, stationary neutral-plasma and radiative transfer models have been used in the interpretation of the data. No stationary VLISM heliospheric model was found that best fitted both the P10 1984 data and the 1986 data. The failure to find a single best fit model is most probably due to the fact that the heliospheric model used here did not incorporate timedependence and interstellar magnetic field effects.
\end{abstract}

\section{Introduction}

The heliosphere a very complicated region surrounding the solar system is shaped by the solar wind, the interstellar plasma, interstellar neutrals, magnetic field, and cosmic rays (Axford, 1972; Holzer, 1972; Zank, 1999; Fahr, 2004; Izmodenov, 2004). It provides a unique opportunity to study in detail the only accessible example of a commonplace but fundamental astrophysical phenomenon - the formation of an astrosphere. The study is made possible through the remote sensing of the VLISM using deep spacecraft extreme ultraviolet (EUV) data. The method uses the measured intensity of the solar Ly $\alpha$ photons scattered by the VLISM neutral hydrogen atoms which penetrate deeply into the heliosphere inspite of the VLISM hydrogen atoms having a strong coupling with the heliospheric plasma protons. The distribution of these atoms inside the heliosphere is influenced by its journey through the interface between the heliosphere and the VLISM. Thus, interstellar hydrogen atoms provide excellent remote diagnostics on the structure of the heliospheric interface and of the heliosphere in general.

Correspondence to: P. Gangopadhyay (pradip@usc.edu)
The photometer on-board P10 has measured the interplanetary Ly $\alpha$ background radiation for more than twenty years and has made a quantitative study of the downstream neutral hydrogen density possible. This analysis uses the latest state of the art neutral hydrogen-plasma and radiative transfer models outlined in the later sections to calculate Ly $\alpha$ glow intensities and these intensities are compared with the P10 data. The new results are presented and the implications discussed.

\section{H atom distribution model}

The distribution of interstellar neutral $\mathrm{H}$ atoms inside the heliosphere is affected by the interaction of the solar wind with the interstellar medium. The modeling of the $\mathrm{H}$ atom flow in the heliosphere is difficult because of its kinetic character due to the large, i.e. comparable to the size of the heliosphere, mean free path due to the charge exchange reaction. To get the $\mathrm{H}$ atom distribution in the heliosphere and the heliospheric interface structure we use a self-consistent model of the solar wind interaction with a two-component interstellar medium. The model is a further development of the BaranovMalama model (Baranov and Malama, 1993). This is an axisymmetric model. Interstellar and interplanetary magnetic fields as well as anomalous and galactic cosmic rays (ACRs and GCRs) are ignored. Influence of ACRs and GCRs on the penetration of the interstellar $\mathrm{H}$ atoms through the interface is insignificant (e.g. Myasnikov et al., 2000, Alexashov et al., 2004). Interstellar magnetic field leads to 3-5\% difference between directions of interstellar $\mathrm{H}$ and $\mathrm{He}$ atom flows in the heliosphere (Lallement et al., 2005; Izmodenov et al., 2005). In the model employed here, the kinetic equation for the neutral component and the hydrodynamic Euler equations were solved self-consistently. Plasma and neutral components interact mainly by charge exchange. However, photoionization, electron impact ionization, solar gravity and solar radiation pressure have also been taken into account. 
Formula for the charge exchange cross section (Maher and Tinsley, 1977) is given below:

$\sigma_{H^{+}, H}(g)=(16.4-1.6 \log (g))^{2} 10^{-16}$,

where $\sigma_{H^{+}, H}$ is in $\mathrm{cm}^{2}$ and $g$ is the relative velocity of atom and proton in $\mathrm{cm} / \mathrm{s}$. Photoionization rate, $\nu_{p h}$, is assumed to be isotropic and is given by rate $\nu_{p h}=\nu_{p h, e}\left(r_{e} / r\right)^{2}$, where $\nu_{p h, e}$, the photoinization rate at the Earth orbit, is given by $1.67 \cdot 10^{-7}$. Voronov (1997) formula for electron impact ionization rate was adopted. Ratio of solar gravity to solar radiation pressure was assumed to be 0.99 .

Basic results of the stationary axially symmetric model were reported by Baranov and Malama (1995), Izmodenov et al. (1999), Izmodenov (2000), Izmodenov et al. (2001), Izmodenov et al. (2003a) and Izmodenov (2003, 2004). The model employed includes both interstellar helium ions and solar wind alpha particles accordingly with as in Izmodenov et al. (2003). The following solar wind boundary conditions were used: (a) number density of solar wind protons and alpha particles of $7.39 \mathrm{~cm}^{-3}$ and $0.185 \mathrm{~cm}^{-3}$, respectively and (b) solar wind velocity of $432 \mathrm{~km} / \mathrm{s}$. The following interstellar neutral parameters used were: (a) interstellar velocity of $26.4 \mathrm{~km} / \mathrm{s}$, (b) neutral temperature of $6519 \mathrm{~K}$ and (c) a ratio of total $\mathrm{H}$ to total $\mathrm{He}$ density, $\left(\mathrm{n}_{H, L I C}+\mathrm{n}_{p, L I C}\right) /\left(\mathrm{n}_{H e, L I C}+\mathrm{n}_{H e^{+}, L I C}\right)$, of 10 . Number density of interstellar $\mathrm{He}$ atoms was assumed to be $0.015 \mathrm{~cm}^{-3}$.

\section{Radiative transfer model}

A Monte Carlo radiative transfer code (Gangopadhyay et al., $1989,2002)$ has been used for this work. It incorporates the self-reversed solar Ly $\alpha$ line, multiple scattering, angle dependent redistribution function, varying hydrogen density and temperature and Doppler effect. A constant solar Ly $\alpha$ line shape (Lemaire et al., 1978) was used. The angle dependent redistribution function (Mihalas, 1978) used here assumes a spherical Maxwellian velocity distribution function. The Doppler effect has to be taken into account since the solar photon frequency and direction are calculated in the stationary sun centered frame while the neutral hydrogen atoms have a position dependent bulk flow velocity relative to the stationary frame.

\section{Instrumentation and data}

The P10 instrument is a two channel photometer that uses the transmission properties and photoelectron response of materials to measure intensities in two spectral regions. A detailed description of the P10 UV photometer is given in Carlson and Judge (1974). The P10 photometer look angle traces out a conical shell (apex angle 40 degrees and shell thickness 1 degree) about the spacecraft spin axis pointing approximately in the direction of the Earth.

We have used 21 P10 daily averaged and look angle averaged Lyman Alpha data obtained in early 1984 and 21 more data obtained in early 1986 at heliospheric distance 31 and $37 \mathrm{AU}$, respectively. P10 data greater than $30 \mathrm{AU}$ was chosen in order to ensure that the modulation of the hydrogen density due to the solar cycle variation of the radiative flux (Blum et al., 1993; Kyrola et al., 1994; Rucinski and Bzowski, 1995) will not significantly affect the results presented here since the deviations of the glow intensity from the stationary model glow intensity are negligible (less than 3\%) (Rucinski and Bzowski, 1995) beyond $30 \mathrm{AU}$ in the downwind direction. We selected P10 data well shortwards of $40 \mathrm{AU}$ in order to stay away from the time when the P10 photometer suffered gain loss (Hall et al., 1993). The ecliptic latitude of P10 was 3.3, and 2.4 degrees and the ecliptic longitude 66 and 68 degrees in early 1984 and early 1986 (Table 1), respectively. P10 was downwind with respect to the interstellar flow and the look directions for all the selected data points in 1984 sampled the downwind direction approximately 139 to 179 degrees and in 1986 from 141 to 180 degrees from the galactic center.

The solar Ly $\alpha$ flux values used in the calculation were obtained from the website http://spacewx.com (Woods et al., 2000). The solar Ly $\alpha$ intensities given in the web site are mostly actual measurements although SME measurements have been rescaled to match the SUSIM UARS calibration and the He $10830 \AA$ has been used as a proxy to fill in some gaps. The solar Ly $\alpha$ flux values used here have been obtained by averaging the daily data over a month to take into account the fact that P10 would see only an average illumination due to their being at large heliocentric distances. This is because in the outer heliosphere there is almost no contribution to the interplanetary glow (Quemerias et al., 2003) from photons coming straight from the sun due to the multiple scattering effect. This means that the entire solar surface and not simply the region facing the spacecraft contributes to the interstellar glow. This averaging effect has also been clearly seen in 1982 when a strong solar cycle effect was damped (Shemansky et al., 1984; Quemerais et al., 1996) at the position of P10.

\section{Comparison of calculations to observations}

Monte Carlo radiative transfer calculations were carried out for various neutral hydrogen density models. The calculated results for the various neutral density models were then compared with the P10 EUV data. It was necessary to calculate the optimum P10 calibration factors (CFs) for each of the density models because of the well known calibration differences between the P10 and V2 spacecraft detectors at Ly $\alpha$ (Shemansky et al., 1984). The calibration factor for a particular density model is obtained by minimizing the least squares sum $L S S$ given by the following equation

$L S S=\sum\left(\left(I_{\text {model }}-C F * I_{\text {space }}\right) / \sigma\right)^{2}$

Where $I_{\text {model }}$ is the calculated intensity, $I_{\text {space }}$ is the measured intensity, $\sigma$ is the standard error for each data point and summation is over the P10 data points for a particular year. 
Table 1. Spacecraft position, look direction, observed intensity and solar flux

\begin{tabular}{|c|c|c|c|c|c|c|c|c|c|c|}
\hline $\mathrm{a}$ & $\mathrm{b}$ & $\mathrm{c}$ & d & $\mathrm{e}$ & $\mathrm{f}$ & $\mathrm{g}$ & $\mathrm{h}$ & $\mathrm{i}$ & $\mathrm{j}$ & 1 \\
\hline P10 & 1984 & 1 & 31.81 & 3.355 & 66.974 & $\mathrm{k}$ & $\mathrm{k}$ & 36.9 & $4.31 \mathrm{E}+11$ & $\mathrm{~m}$ \\
\hline P10 & 1984 & 4 & 31.82 & 3.340 & 66.912 & $\mathrm{k}$ & $\mathrm{k}$ & 37.0 & $4.30 \mathrm{E}+11$ & $\mathrm{~m}$ \\
\hline P10 & 1984 & 7 & 31.84 & 3.324 & 66.850 & $\mathrm{k}$ & $\mathrm{k}$ & 36.8 & $4.31 \mathrm{E}+11$ & $\mathrm{~m}$ \\
\hline P10 & 1984 & 10 & 31.86 & 3.309 & 66.789 & $\mathrm{k}$ & $\mathrm{k}$ & 37.2 & $4.33 \mathrm{E}+11$ & $\mathrm{~m}$ \\
\hline P10 & 1984 & 13 & 31.88 & 3.305 & 66.730 & $\mathrm{k}$ & $\mathrm{k}$ & 36.9 & $4.36 \mathrm{E}+11$ & $\mathrm{~m}$ \\
\hline P10 & 1984 & 16 & 31.90 & 3.308 & 66.673 & $\mathrm{k}$ & $\mathrm{k}$ & 36.8 & $4.40 \mathrm{E}+11$ & $\mathrm{~m}$ \\
\hline P10 & 1984 & 19 & 31.93 & 3.310 & 66.616 & $\mathrm{k}$ & $\mathrm{k}$ & 37.2 & $4.44 \mathrm{E}+11$ & $\mathrm{~m}$ \\
\hline P10 & 1984 & 22 & 31.95 & 3.313 & 66.559 & $\mathrm{k}$ & $\mathrm{k}$ & 37.8 & $4.46 \mathrm{E}+11$ & $\mathrm{~m}$ \\
\hline P10 & 1984 & 25 & 31.97 & 3.316 & 66.502 & $\mathrm{k}$ & $\mathrm{k}$ & 38.1 & $4.48 \mathrm{E}+11$ & $\mathrm{~m}$ \\
\hline P10 & 1984 & 28 & 31.99 & 3.318 & 66.445 & $\mathrm{k}$ & $\mathrm{k}$ & 38.1 & $4.52 \mathrm{E}+11$ & $\mathrm{~m}$ \\
\hline P10 & 1984 & 31 & 32.01 & 3.321 & 66.388 & $\mathrm{k}$ & $\mathrm{k}$ & 37.8 & $4.58 \mathrm{E}+11$ & $\mathrm{~m}$ \\
\hline $\mathrm{P} 10$ & 1984 & 34 & 32.04 & 3.321 & 66.346 & $\mathrm{k}$ & $\mathrm{k}$ & 37.7 & $4.61 E+11$ & $\mathrm{~m}$ \\
\hline P10 & 1984 & 37 & 32.06 & 3.316 & 66.334 & $\mathrm{k}$ & $\mathrm{k}$ & 37.8 & $4.63 \mathrm{E}+11$ & $\mathrm{~m}$ \\
\hline P10 & 1984 & 40 & 32.08 & 3.311 & 66.322 & $\mathrm{k}$ & $\mathrm{k}$ & 38.2 & $4.65 E+11$ & $\mathrm{~m}$ \\
\hline P10 & 1984 & 43 & 32.11 & 3.307 & 66.310 & $\mathrm{k}$ & $\mathrm{k}$ & 37.9 & $4.68 \mathrm{E}+11$ & $\mathrm{~m}$ \\
\hline P10 & 1984 & 46 & 32.13 & 3.302 & 66.298 & $\mathrm{k}$ & $\mathrm{k}$ & 37.5 & $4.74 \mathrm{E}+11$ & $\mathrm{~m}$ \\
\hline P10 & 1984 & 49 & 32.15 & 3.303 & 66.298 & $\mathrm{k}$ & $\mathrm{k}$ & 38.1 & $4.78 \mathrm{E}+11$ & $\mathrm{~m}$ \\
\hline P10 & 1984 & 52 & 32.18 & 3.303 & 66.299 & $\mathrm{k}$ & $\mathrm{k}$ & 37.9 & $4.80 \mathrm{E}+11$ & $\mathrm{~m}$ \\
\hline P10 & 1984 & 55 & 32.20 & 3.304 & 66.299 & $\mathrm{k}$ & $\mathrm{k}$ & 37.0 & $4.78 \mathrm{E}+11$ & $\mathrm{~m}$ \\
\hline P10 & 1984 & 58 & 32.22 & 3.303 & 66.297 & $\mathrm{k}$ & $\mathrm{k}$ & 36.9 & $4.75 E+11$ & $\mathrm{~m}$ \\
\hline P10 & 1984 & 61 & 32.24 & 3.301 & 66.293 & $\mathrm{k}$ & $\mathrm{k}$ & 38.1 & $4.75 \mathrm{E}+11$ & $\mathrm{~m}$ \\
\hline P10 & 1986 & 1 & 37.28 & 2.400 & 68.553 & $\mathrm{k}$ & $\mathrm{k}$ & 25.5 & $3.68 \mathrm{E}+11$ & $\mathrm{~m}$ \\
\hline P10 & 1986 & 4 & 37.31 & 2.407 & 68.547 & $\mathrm{k}$ & $\mathrm{k}$ & 25.5 & $3.67 \mathrm{E}+11$ & $\mathrm{~m}$ \\
\hline P10 & 1986 & 7 & 37.33 & 2.413 & 68.540 & $\mathrm{k}$ & $\mathrm{k}$ & 25.5 & $3.66 \mathrm{E}+11$ & $\mathrm{~m}$ \\
\hline P10 & 1986 & 10 & 37.35 & 2.418 & 68.536 & $\mathrm{k}$ & $\mathrm{k}$ & 24.9 & $3.66 \mathrm{E}+11$ & $\mathrm{~m}$ \\
\hline P10 & 1986 & 13 & 37.38 & 2.423 & 68.534 & $\mathrm{k}$ & $\mathrm{k}$ & 24.8 & $3.67 \mathrm{E}+11$ & $\mathrm{~m}$ \\
\hline P10 & 1986 & 16 & 37.40 & 2.424 & 68.533 & $\mathrm{k}$ & $\mathrm{k}$ & 25.4 & $3.68 \mathrm{E}+11$ & $\mathrm{~m}$ \\
\hline P10 & 1986 & 19 & 37.42 & 2.414 & 68.536 & $\mathrm{k}$ & $\mathrm{k}$ & 25.1 & $3.69 \mathrm{E}+11$ & $\mathrm{~m}$ \\
\hline P10 & 1986 & 22 & 37.44 & 2.405 & 68.541 & $\mathrm{k}$ & $\mathrm{k}$ & 24.9 & $3.71 \mathrm{E}+11$ & $\mathrm{~m}$ \\
\hline P10 & 1986 & 25 & 37.46 & 2.396 & 68.548 & $\mathrm{k}$ & $\mathrm{k}$ & 25.2 & $3.73 \mathrm{E}+11$ & $\mathrm{~m}$ \\
\hline P10 & 1986 & 28 & 37.49 & 2.386 & 68.555 & $\mathrm{k}$ & $\mathrm{k}$ & 25.4 & $3.75 \mathrm{E}+11$ & $\mathrm{~m}$ \\
\hline P10 & 1986 & 31 & 37.51 & 2.377 & 68.553 & $\mathrm{k}$ & $\mathrm{k}$ & 25.5 & $3.78 \mathrm{E}+11$ & $\mathrm{~m}$ \\
\hline P10 & 1986 & 34 & 37.53 & 2.370 & 68.531 & $\mathrm{k}$ & $\mathrm{k}$ & 25.4 & $3.80 \mathrm{E}+11$ & $\mathrm{~m}$ \\
\hline P10 & 1986 & 37 & 37.55 & 2.376 & 68.539 & $\mathrm{k}$ & $\mathrm{k}$ & 25.1 & $3.80 \mathrm{E}+11$ & $\mathrm{~m}$ \\
\hline P10 & 1986 & 40 & 37.58 & 2.389 & 68.563 & $\mathrm{k}$ & $\mathrm{k}$ & 25.2 & $3.80 \mathrm{E}+11$ & $\mathrm{~m}$ \\
\hline P10 & 1986 & 43 & 37.60 & 2.395 & 68.579 & $\mathrm{k}$ & $\mathrm{k}$ & 25.1 & $3.88 \mathrm{E}+11$ & $\mathrm{~m}$ \\
\hline P10 & 1986 & 46 & 37.62 & 2.387 & 68.579 & $\mathrm{k}$ & $\mathrm{k}$ & 25.4 & $3.89 \mathrm{E}+11$ & $\mathrm{~m}$ \\
\hline P10 & 1986 & 49 & 37.64 & 2.380 & 68.579 & $\mathrm{k}$ & $\mathrm{k}$ & 25.7 & $3.90 \mathrm{E}+11$ & $\mathrm{~m}$ \\
\hline P10 & 1986 & 52 & 37.67 & 2.384 & 68.580 & $\mathrm{k}$ & $\mathrm{k}$ & 25.2 & $3.91 \mathrm{E}+11$ & $\mathrm{~m}$ \\
\hline P10 & 1986 & 55 & 37.69 & 2.388 & 68.581 & $\mathrm{k}$ & $\mathrm{k}$ & 25.9 & $3.92 \mathrm{E}+11$ & $\mathrm{~m}$ \\
\hline P10 & 1986 & 58 & 37.71 & 2.392 & 68.582 & $\mathrm{k}$ & $\mathrm{k}$ & 25.8 & $3.91 \mathrm{E}+11$ & $\mathrm{~m}$ \\
\hline P10 & 1986 & 61 & 37.73 & 2.396 & 68.583 & $\mathrm{k}$ & $\mathrm{k}$ & 26.1 & $3.91 \mathrm{E}+11$ & $\mathrm{~m}$ \\
\hline
\end{tabular}

$\mathrm{a}=$ Spacecraft $; \mathrm{b}=$ Year $\mathrm{c}=$ day $; \mathrm{d}=$ heliocentric distance $(\mathrm{AU}) ; \mathrm{e}=$ Sun centered ecliptic latitude of the spacecraft; $\mathrm{f}=$ Sun centered ecliptic longitude of the spacecraft; $g=$ Sun centered ecliptic latitude of the look direction; $h=$ Sun centered ecliptic longitude of the look direction; $\mathrm{i}=$ intensity in Rayleighs; $\mathrm{j}=$ solar flux in photons $\mathrm{cm}^{-2} \mathrm{~s}^{-1} ; \mathrm{k}=\mathrm{P} 10$ look direction is described in the text; $1=$ angle between look direction and galactic center; $\mathrm{m}=$ angle between $\mathrm{P} 10$ look angle and galactic center direction is discussed in the text.

We have assumed a zero Galactic Ly $\alpha$ background since, except possibly for the region close to the Galactic center, the Galactic Ly $\alpha$ background has been found to be small (Shemansky et al., 1984; Gangopadhyay et al., 2002; Quemerais et al., 2003; Gangopadhyay et al., 2005). The standard error $\sigma$ is given by the following equation:

$\sigma=I \sqrt{\left(\frac{\delta n}{n}\right)^{2}+\left(\frac{\delta F}{F}\right)^{2}}$ where $I$ is the calculated intensity, $\delta n / n$ is the fractional uncertainty in the number of photons, $n$, collected by the detector and $\delta F / F$ is the fractional uncertainty in the solar flux, $F$, used here. $\delta n / n$ is given by $1 / \sqrt{(} n)$. The fractional uncertainty in the solar flux, estimated from the standard deviation of the daily solar flux during P10 observations, was found to be $2.5 \%$ in 1984 and $3 \%$ in 1986 . Thus $\delta F / F$ was set equal to 0.025 for the $1984 \mathrm{P} 10$ data and set equal to 0.03 for the 1986 P10 data. 
Table 2. Sets of model parameters and results

\begin{tabular}{cccccc}
\hline $\begin{array}{c}\text { Space- } \\
\text { craft }\end{array}$ & year & $\begin{array}{c}\text { H Density } \\
\left(\mathrm{cm}^{-3}\right)\end{array}$ & $\begin{array}{c}\mathrm{H}^{+} \text {Density } \\
\left(\mathrm{cm}^{-3}\right)\end{array}$ & $\sqrt{ }(L S S)$ & $\mathrm{CF}$ \\
\hline P10 & 1984 & 0.15 & 0.05 & 0.25 & 2.36 \\
P10 & 1984 & 0.15 & 0.07 & 0.29 & 2.20 \\
P10 & 1984 & 0.18 & 0.06 & 0.44 & 2.74 \\
P10 & 1984 & 0.20 & 0.10 & 0.43 & 2.59 \\
P10 & 1986 & 0.15 & 0.05 & 0.21 & 2.86 \\
P10 & 1986 & 0.15 & 0.07 & 0.27 & 2.60 \\
P10 & 1986 & 0.18 & 0.06 & 0.34 & 2.89 \\
P10 & 1986 & 0.20 & 0.10 & 0.27 & 2.98 \\
\hline
\end{tabular}

The $L S S$ is a measure of the fit of the calculated results with the P10 data, the smaller the $L S S$ better the fit. The procedure of minimization of $L S S$ discriminates between the different functional dependences of the glow on look angles and heliocentric distances and thus different heliospheric models since heliospheric models with different hydrogen and proton densities will yield backscattered glow with different functional dependences on look angles and heliocentric distances. The $\sqrt{(} L S S)$, CF for P10 are given in Table 2. It is clear from Table 2 that none of the four VLISM models best fits the P10 data taken at two different times. The VLISM model with a neutral hydrogen density of $0.15 \mathrm{~cm}^{-3}$ and proton density of $0.05 \mathrm{~cm}^{-3}$ fitted both the P10 1984 and 1986 data better than other models. However, two different CFs had to be used in order to get the fit to the data in 1984 and 1986. The use of two CFs does not necessarily imply that the calibration of P10 has changed between early 1984 and early 1986. It means that the functional dependence of the observed glow on look angles and heliocentric distances did not change and that the only thing that changed between early 1984 and early 1986 was the absolute value of the glow along the P10 line of sight. This result is consistent with the fact that P10 look angle data were flat to within less than 5\% (Wu et al., 1988) during this time period. We have also investigated the calibration change issue by comparing two V2 and P10 observations in 1984 and 1985. P10 photometer observed 31.2 and 27.9 Rayleighs in 1984, day 331 and 1985, day 331 at heliocentric distances of 34.28 and 37.028 AU, respectively. V2 observed 387 and 290 Rayleighs on the same days at heliocentric distances of 15.6 AU and 18.7 AU, respectively. The ratio of V2 intensity multiplied by distance to P10 intensity multiplied by its heliocentric distance yielded 5.6 and 5.2 on these two days. The ratios, after taking into account the change in look direction between these two days and the fact that the spacecraft in situ solar flux values are not exactly known, appear to be nearly constant suggesting that P10 photometer did not suffer any gain loss during this period. The P10 1984 and 1986 data as modified by the appropriate CFs and the calculated intensities are shown in Figs. 1 and 2 for the VLISM heliospheric models with a neutral hydrogen density of $0.15 \mathrm{~cm}^{-3}$ and proton density of $0.05 \mathrm{~cm}^{-3}$. The results obtained here can not be compared with previous calculations (Gangopad-

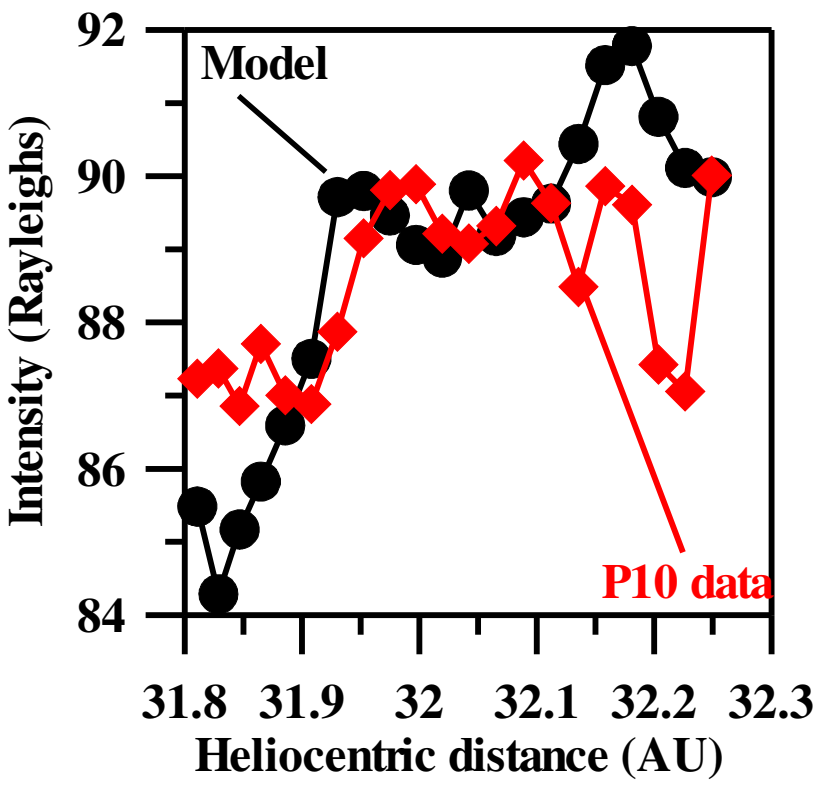

Fig. 1. The calculated intensity for the heliospheric model with neutral hydrogen density of $0.15 \mathrm{~cm}^{-3}$ and proton density of 0.05 $\mathrm{cm}^{-3}$ and P10 intensity modified by a calibration factor of 2.36 are plotted against spacecraft heliocentric distance. The standard error for the calculated intensity in this and subsequent figures is calculated by the equation given in the text.

hyay et al., 2002, 2004, 2005; Izmodenov et al., 2003b) since those calculations used spacecraft data spread over an entire solar cycle.

\section{Discussion and Conclusion}

One possible reasons for our failure to find a single heliospheric plasma-neutral model fitting both the 1984 and 1986 P10 data could be that we did not choose the right VLISM heliospheric model since we tested only a few VLISM models with different neutral hydrogen and proton densities. Another possibility is that we are seeing time-dependent effects. It is of course certain that a time-dependent heliosphere model will be necessary to fully interpret the glow data because there is a definite possibility that the heliosphere is "breathing in or out" due to pressure fluctuations caused by the well-known solar cycle variations. In this case there will be regions of high neutral hydrogen density followed by regions of lower density because the amount of VLISM neutral hydrogen filtering through the interface will change over an entire solar cycle and will cause the neutral hydrogen density inside the heliosphere to fluctuate. This fluctuation in neutral hydrogen density would show up as different values of $\mathrm{CF}$ when a stationary model is used. The $\mathrm{P} 10$ result suggests that the absolute value of the neutral hydrogen density in the heliosphere downstream region was different in 1984 compared to the downstream neutral hydrogen density in 1986 and this difference is due to the change in the amount of the filtration of neutral hydrogen atoms at the heliospheric interface many years before the P10 observation. The results 


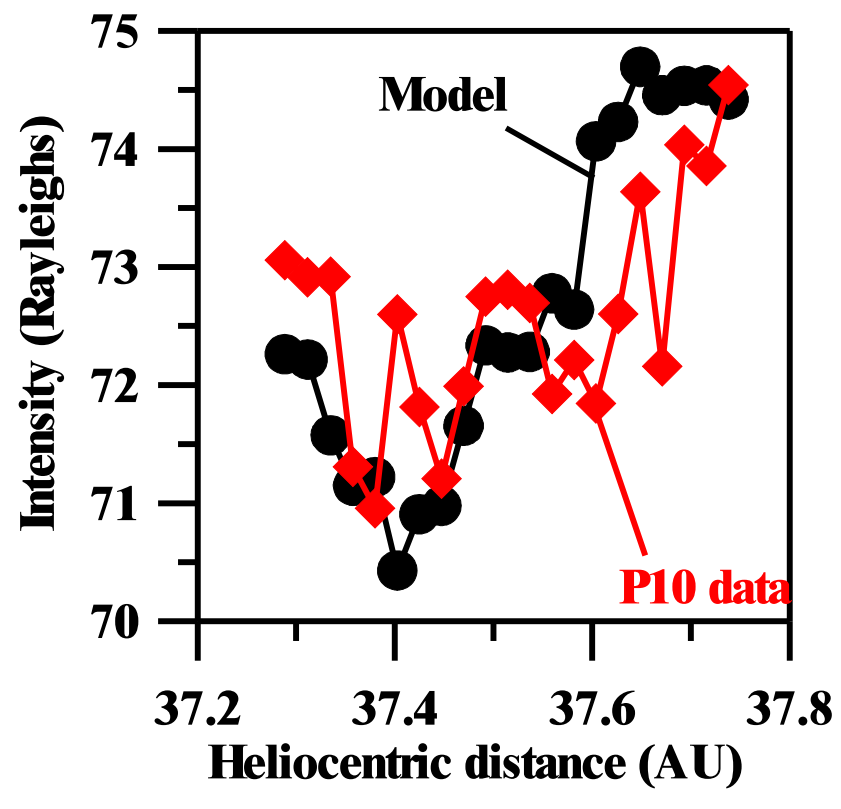

Fig. 2. The calculated intensity for the heliospheric model with neutral hydrogen density of $0.15 \mathrm{~cm}^{-3}$ and proton density of 0.05 $\mathrm{cm}^{-3}$ and $\mathrm{P} 10$ intensity modified by a calibration factor of 2.86 are plotted against spacecraft heliocentric distance.

presented here suggest the need to use time-dependent heliosphere models. A third possibility is that the squeezing of the heliospheric interface due to the interstellar magnetic field is being seen since there is no doubt that magnetic fields affect the heliospheric neutral hydrogen density. A model like that of Izmodenov et al., 2005 that incorporates the interstellar magnetic field and uses a kinetic treatment of the neutral $\mathrm{H}$ atom flow should be used in future for the interpretation of the glow data.

Solar Ly $\alpha$ line shape variations may also need to be taken into account. It is well known that photons from the solar Ly $\alpha$ line core and not the wings resonantly scatter from the inflowing interstellar hydrogen atoms. What is measured, however, is the solar flux integrated over the whole line and not the core. The fraction of core photons will vary with the solar cycle if there is any solar cycle line shape variation. We have in this calculation assumed a fixed line shape which may not hold over the entire solar cycle (Lemaire et al., 1998). A photon redistribution function that uses the actual $\mathrm{H}$ distribution function and not a spherical Maxwellian distribution function may need to be taken into account.

The change in CF, about 15-20\%, between early 1984 and early 1986 is a measure of the change in the absolute value of glow and hence of the change of the parameters (density and temperature) of the neutral hydrogen density along the P10 line of sight. How does this compare with the various model predictions published in the literature? There are currently three time-dependent heliospheric models available (Zank and Muller, 2003; Scherer and Fahr, 2003a,b; and Izmodenov et al., 2005). The first two models use a multifluid treatment for the inflowing neutral $\mathrm{H}$ atoms. Alexashov and Izmodenov (2005) have shown significant difference in the results of kinetic and multi-fluid models in the stationary case. Izmodenov et al. (2005) have also found significant difference between the results of their kinetic model and the results of multi-fluid models in the non-stationary case. Therefore, using multi-fluid model for interpretation of observational data may lead to incorrect conclusion. It should also be pointed out that a comparison between the multi-fluid model predictions and the results obtained here would require detailed radiative transfer calculations using the multifluid neutral $\mathrm{H}$ distributions which is outside the scope of this paper. A detailed calculation would be necessary because the glow intensity depends in a complicated manner on the density, velocity and temperature of the neutral $\mathrm{H}$ distribution.

Izmodenov et al. (2005) using a kinetic treatment for the inflowing neutral $\mathrm{H}$ atoms found a solar cycle induced 10$12 \%$ fluctuation in the neutral hydrogen density for both the primary and the secondary interstellar $\mathrm{H}$ atoms created in the heliosheath at large heliocentric distances. This result is in qualitative agreement with the results obtained here. A quantitative agreement is not expected since Izmodenov et al. (2005) say that their results cannot be directly applied to interpretation of observational data. The results presented here are in qualitative agreement with Quemerais et al. (2003). Quemerais et al. (2003), however, saw the coefficient of the power law describing the glow intensity as a function of solar distance change from -1.58 between 1993 to 1997 to a value of -0.22 after 1998 . P10 did not see any such change.

\section{Acknowledgement}

This work was supported by NASA grant NNG04GB80G. It is a pleasure to acknowledge the computational support given by the University of Southern California High Performance Computing and Communication (HPCC) center. V.I. was supported in part by INTAS Award 2001-0270, RFBR grants 04-02-16559, 04-01-00594, RFBR-GFEN grant 0301-39004, and International Space Science Institute in Bern. Edited by: H.-J. Fahr

Reviewed by: two referees

\section{References}

Alexashov, D. B., Chalov, S. V., Myasnikov, A. V., Izmodenov, V. V., Kallenbach, R.: The dynamical role of anomalous cosmic rays in the outer heliosphere, Astron. Astrophys. 420, 729-736, 2004.

Axford, W. I.: The interaction of the solar wind with the interstellar medium, Solar Wind, NASA Spec. Publ., NASA SP-308, 609-658, 1972.

Baranov, V. B. and Malama, Y. G.: Model of the solar wind interaction with the local interstellar medium - Numerical solution of self-consistent problem, J. Geophys. Res., 98, 5 157-15163, 1993.

Baranov, V. B. and Malama, Y. G.: Effect of local interstellar medium hydrogen fractional ionization on the distant solar wind and interface region, J. Geophys Res., 100, 14 755-14 762, 1995. 
Blum, P., Gangopadhyay, P., Ogawa, H. S., and Judge, D. L.: Solar driven neutral density wave, Astron. Astrophys., 272, 549-554, 1993.

Carlson, R. W. and Judge, D. L.: Pioneer 10 Ultraviolet photometer observations at Jupiter encounter, J. Geophys. Res., 79, 3623-3633, 1974.

Fahr, H. J.: Global structure of the heliosphere and interaction with the local interstellar medium: three decades of growing knowledge, Adv. Space Res., 34, 3-13, 2004.

Gangopadhyay, P., Izmodenov, V. V., Gruntman, M., and Judge, D. L.: Interpretation of Pioneer 10 Ly $\alpha$ based on heliospheric interface models: methodology and first results, J. Geophys. Res., 107, 17-1, doi:10.1029/2002JA009345, 2002.

Gangopadhyay, P., Izmodenov, V. V., Quemerais, E., Gruntman, M., and Judge, D. L.: Interpretation of Pioneer 10 and Voyager 2 Ly $\alpha$ Data: First Results, Adv. Space Res., 34, 1, 94-98, 2004.

Gangopadhyay, P., Izmodenov, V. V., Shemansky, D. E., Gruntman, M., and Judge, D. L.: Reappraisal of the Pioneer 10 and Voyager 2 Ly $\alpha$ intensity measurements, Astrophys. J., 628, 514-519, 2005.

Gangopadhyay, P., Ogawa, H. S., Judge, D. L.: Evidence of a nearby solar wind shock as obtained from distant Pioneer 10 ultraviolet glow data, Astrophys. J., 336, 1012-1021, 1989.

Hall, D., Shemansky, D. E., Judge, D. L., Gangopadhyay, P., and Gruntman, M. A.: Heliospheric hydrogen beyond $15 \mathrm{AU}$ : Evidence for a termination shock, J. Geophys. Res., 98, 15 185-15 192, 1993.

Holzer, T. E.: Interaction of the solar wind with the neutral component of the interstellar gas, J. Geophys. Res., 77, 5407-5431, 1972.

Izmodenov, V. V.: Physics and Gasdynamics of the Heliospheric Interface, Astrophys. Space Science, 274, 55-69, 2000.

Izmodenov, V. V.: Models of Solar wind interaction with the local interstellar cloud, in Proc. of the "Interstellar environment of the heliosphere" COSPAR colloquium in honor of Stanislaw Grzedzielski, edited by Breitschwerdt, D. and Haerendel, G., MPE Report, 285, 113-134, 2003.

Izmodenov, V. V.: The heliospheric interface: models and observations, Chapter in "The Sun and the heliosphere as an integrated system", edited by Poletto, G. and Suess, S. T., Kluwer, 317, 23-64, 2004.

Izmodenov, V. V., Alexashov, D., and Myasnkov, A.: Direction of the interstellar $\mathrm{H}$ atom inflow in the heliosphere: role of the interstellar magnetic field, Astron. Asrophys., 437, L35-L38, 2005.

Izmodenov, V. V., Gangopadhyay, P., Quemerais, E., Gruntman, M., and Judge, D. L.: Interstellar Pioneer 10 EUV data: Possible constraints on the local interstellar parameters, Solar Wind Ten:, Proc. of the Tenth International Solar Wind Conference, edited by Velli, M. and Bruno, R., AIP Conf. Proceed., 679, 198-201, $2003 \mathrm{~b}$.

Izmodenov, V. V., Gruntman, M., and Malama, Y. G.: Interstellar hydrogen atom distribution function in the outer heliosphere, J. Geophys. Res., 106, 10 681-10 690, 2001.

Izmodenov, V. V., Lallement, R., and Malama, Y. G.: Heliospheric and astrospheric hydrogen absorption towards Sirius: no need for interstellar hot gas, Astron. Astrophys., 342, 13-16, 1999.

Izmodenov, V. V., Malama, Yu. G., Gloeckler, G., and Geiss, J.: Effects of interstellar and solar wind ionized helium on the interaction of the solar wind with the local interstellar medium, Astrophys. J., Letters, 954, L59-L62, 2003a.
Izmodenov, V. V., Malama, Yu. G., and Ruderman, M.: Solar cycle influence on the interaction of the solar wind with Local Interstellar Cloud, Astron. Astrophys., 429, 1069-1080, 2005.

Kyrola, E., Summanen, T., and Raback, P.: Solar cycle and interplanetary hydrogen, Astron. Astrophys., 288, 299-314, 1994.

Lallement, R., Que'merais, E., Bertaux, J. L., Ferron, S., Koutroumpa, D., and Pellinen, R.: Deflection of the Interstellar Neutral Hydrogen Flow Across the Heliospheric Interface, Science, 307, 1447-1449, 2005.

Lemaire, P., Charra, J., Jouchox, A., Vidal-Madjar, A., Artzner, G. E., Vial, J. C., Bonnet. R. M., Skumanich, A., Schuhle, U., and Wilhelm, K.: Solar HI Lyman $\alpha$ full disk profile obtained with SUMER/SOHO spectrometer, Astrophys. J., 223, L55-L58, 1978.

Maher, L. J. and Tinsley, B. A.: Atomic hydrogen escape rate due to charge exchange with hot plasmaspheric ions, J. Geophys. Res., 82, 689-695, 1977.

Miahalas, D.: Stellar Atmospheres, W. H. Freeman and comp., 415-419, 1978.

Myasnikov, A. V., Alexashov, D. B., Izmodenov, V. V., and Chalov, S. V.: Self-consistent model of the solar wind interaction with three-component circumsolar interstellar cloud: Mutual influence of thermal plasma and galactic cosmic rays and $\mathrm{H}$-atoms, J. Geophys. Res., 105, 5167-5168, 2000.

Quemerais, E., Bertaux, J.-L., Lallement, R., Sandel, B. R., and Izmodenov, V. V.: Voyager 1/UVS Ly $\alpha$ glow data from 1993 to 2003: the hydrogen distribution in the upwind outer heliosphere, J. Geophys. Res., 108, 4-1, doi:10.1029/2003JA009871, 2003.

Quemerais, E., Sandel, B. R., and Toma, G. D.: 26 day modulation of the sky background Ly $\alpha$ brightness: estimating the interplanetary hydrogen density, Astrophys. J., 463, 349-358, 1996.

Rucinski, D. and Bzowski, M.: Breathing of the heliospheric structures triggered by the solar cyclic activity, Astron. Astrophys, 296, 248-263, 1995.

Shemansky, D. E., Judge, D. L., and Jessen, J. M.: Pioneer 10 and Voyager observations of the interstellar medium in scattered emission of the He 584 A and Ly $\alpha 1216$ A lines in NASA Goddard Space Flight Center, Local Interstellar Medium, 81, 24-27, 1984.

Scherer, K. and Fahr, H. J.: Solar cycle variations in the outer heliosphere, Geophys. Res. Lett., 30, 2, 17-1, doi:10.1029/2002GL016073, 2003a.

Scherer, K. and Fahr, H. J.: Breathing of the heliospheric structures triggered by the solar cyclic activity, Annales Geophys., 21, 1303-1313, 2003b.

Voronov, G. S.: Atom. Data Nucl. Data Tables, 65, 1-35, 1997.

Woods, T. N., Tobiska, W. K., Rottman, G. J., and Worden, J. R.: Improved solar Ly $\alpha$ irradiance modeling from 1947 through 1999 based on UARS observations, J. Geophys. Res., 105, 27 195-27 215, 2000.

Wu, F. M., Gangopadhyay, P., Ogawa, H. S., and Judge, D. L.: The hydrogen density of the local interstellar medium and an upper limit to the galactic glow determined from Pioneer 10 ultraviolet photometer observations, Astrophys. J., 331, 1004-1012, 1988.

Zank, G. P.: Interaction of the solar wind with the local interstellar medium: a theoretical perspective, Space Sci. Rev., 89, 3-4, 413-688, 1999.

Zank, G. P. and Mueller, H. R.: The dynamical heliosphere, J. Geophys. Res., 108, 7-1, doi:10.1029/2002JA009689, 2003. 